\title{
Originales
}

\section{Estrategias de información en una Unidad de Cuidados Intensivos polivalente}

\author{
R. ABIZANDA CAMPOS ${ }^{a}, A$. BERNAT ADELL ${ }^{a}$, R. BALLESTER ARNAL ${ }^{b}$, E. BISBAL ANDRÉSa, \\ B. VIDAL TEGEDOR ${ }^{a}$, M. CUBEDO BORT'a ${ }^{\mathrm{Y}}$ R. REIG VALERO ${ }^{a}$ \\ aServicio de Medicina Intensiva. Hospital Universitario Asociado General de Castellón. Castellón. España. \\ bDepartamento de Psicología Clínica y Psicobiología. Universidad Jaume I. Castellón. España.
}

\begin{abstract}
Introducción. La comprensión de la información proporcionada a los familiares y allegados de los enfermos críticos ingresados en la Unidad de Cuidados Intensivos (UCI), y la transmisión, adecuada y sin contradicciones, de esta información, es un aspecto fundamental relacionado con la posible participación en las decisiones terapéuticas y con la calidad percibida respecto al proceso asistencial. Con el fin de establecer la adecuación de estos dos aspectos (comprensión y transmisión de la información) se ha realizado el presente estudio.
\end{abstract}

Diseño. Estudio de opinión elaborado por el equipo médico, con la supervisión de enfermería de una $\mathrm{UCI}$ polivalente.

Método. Estudio cualitativo observacional. Encuesta de preguntas de respuesta abierta. Acuerdo de terminología y conceptos que debe contener la información y estimación de los distintos contenidos de información proporcionada por los principales estamentos asistenciales (médicos y enfermeras). Elaboración, por técnica Delphi, de una hoja de transmisión de la información entre distintos facultativos, con el fin de homogeneizar el proceso informativo.

Resultados. El análisis del cuestionario demuestra la gran heterogeneidad de los contenidos y formas de la información proporcionada, lo que repercute en defectos de comprensión y aparentes contradicciones, y a su vez dificulta la com-

\footnotetext{
Correspondencia: R. Abizanda.

Servicio de Medicina Intensiva.

Hospital Universitario Asociado General de Castellón.

Avda. Benicàssim, s/n.

12004 Castellón. España.

Correo electrónico: abizanda_ric@gva.es

Correo electrónico: rabizandac@terra.es

Manuscrito aceptado el 26-X-2007.
}

prensión de esa información y la integración de familiares y allegados en el proceso asistencial.

Se presenta el acuerdo logrado entre los distintos facultativos encuestados para facilitar la información y evitar interpretaciones subjetivas por parte de los informados.

PALABRAS CLAVE: información, política de información, transmisión de la información.

\section{INFORMATION STRATEGIES IN A POLYVALENT INTENSIVE CARE UNIT}

Introduction. Understanding the information provided to families and surrogates of the critically ill patients admitted to ICUs and its adequate communication without contradictions, is a fundament aspect related with the possible participation of these persons in the treatment decision making and with the quality perceived regarding the care process. Our aim in this study is to assess these two aspects (information and communication of information).

Design. Opinion study elaborated by the medical team and nursing staff of a multidisciplinary ICU.

Method. Observational qualitative study performed through an open answers questionnaire. Search for agreement on terminology and concepts that should be included in the information and estimation of the different contents of information provided by the main health care professional groups (physicians and nurses). Using the Delphi technique to elaborate an information communication sheet between different staff members in order to homogenize the information process.

Results. The analysis of the questionnaire reveals the great heterogeneity of the contents and modes of information provided. This may cause 
difficulties in understanding and the integration of families and relatives in the care process.

The agreement achieved among the different between physicians to facilitate the information and avoid subjective interpretations by the informed people is presented.

KEY WORDS: information, information policies, information contents.

\section{INTRODUCCIÓN}

La información es, según el diccionario de la Real Academia Española en su 22. ${ }^{a}$ edición ${ }^{1}$, «un proceso de comunicación o adquisición de conocimientos que permitan ampliar o precisar los que se poseen sobre una materia determinada» (5. ${ }^{\text {a }}$ acepción $)$.

Desde este punto de vista, podemos considerar la información (proceso de relación verbal, en el que se produce un continuo intercambio e interacción de información entre el personal sanitario y el enfermo) como inseparable de la asistencia sanitaria, tanto desde una perspectiva deontológica como ética estricta. La norma, consagrada en los distintos códigos profesionales y en la Ley 41/2002 (que regula la autonomía del paciente $)^{2}$, y la más elemental obligación hacia aquellos que buscan ayuda en el médico (en el sanitario en general), obligan a mantener un proceso de diálogo mediante el cual el profesional comunica al paciente cuáles son las sospechas, expectativas y alternativas de tratamiento asociadas a su estado de salud.

En el ámbito de la medicina intensiva, cuando la competencia del paciente está comprometida, ya sea por la situación patológica per se o como consecuencia de intervenciones terapéuticas (el caso más paradigmático es el de la sedoanalgesia), esta obligación no desaparece, sino que se traslada, proyectándose hacia aquellos que pueden tomar, si es necesario, decisiones encaminadas al mejor beneficio del propio enfermo. Sean estas personas familiares, allegados o representantes legales, el equilibrio entre información y comprensión de la misma es un deber para todos los profesionales implicados.

Sin embargo, esta información tiene los límites impuestos por su adecuado entendimiento, y ello, a su vez, puede estar condicionado por el nivel cultural y de estudios del informado, y por la forma de expresión del informador.

En un estudio previo, nuestro grupo constató un alto nivel de satisfacción de un grupo de familiares ante la información recibida ${ }^{3}$. Pese a ello, existe entre los profesionales de nuestro servicio una profunda preocupación por el hecho de garantizar la comprensión de la información proporcionada, exenta de ambigüedades y malas interpretaciones, y desprovista (en lo posible) de la muy frecuente variabilidad personal. Por este motivo, se ha realizado el presente estudio.

\section{MATERIAL Y MÉTODO}

Con el objetivo de armonizar y establecer un acuerdo sobre los contenidos de la información facilitada a los familiares/allegados de los pacientes ingresados en la Unidad de Cuidados Intensivos (UCI), y partiendo de la base de la falta de competencia de los mismos en más de un $85 \%{ }^{4}$, se administró un cuestionario de preguntas abiertas a un total de 19 personas ( 1 jefe de servicio, 10 adjuntos, 7 MIR y una supervisora). El cuestionario incluía las siguientes preguntas:

1. ¿Qué cosas quisiera saber sobre la familia o allegados a los que informa?

2. ¿Qué considera importante conocer de lo que se ha dicho los días previos?

3. ¿Qué expresiones o términos cree que deberían desterrarse del lenguaje de información?

4. Sugiera una escala de cinco niveles de gravedad/pronóstico adaptada a un lenguaje normal y ordenada de peor a mejor.

5. Sugiera tres contenidos/niveles de información que deban ser obligadamente conocidos por la familia informada.

6. ¿Sobre qué puntos debe la enfermería dar información sobre la situación del paciente? Esta pregunta permitía responder «sí»o «no» a los siguientes puntos: situación del paciente, aclaraciones sobre el tratamiento administrado, situación de gravedad, diagnósticos, pronóstico, planes de actuación y cuidados ofertados.

7. Sugiera el contenido de una hoja de transmisión de información sobre la información facilitada por otro médico.

8. Otras sugerencias.

En conjunto se recogieron los 19 cuestionarios administrados y el análisis fue realizado por un subgrupo de la misma muestra encuestada.

\section{RESULTADOS}

El primer hallazgo que debe destacarse es la gran dispersión de respuestas, lo que obliga a una presentación esquemática de los resultados, que se presentan en las tablas 1,2 y 3 . Debe tenerse en cuenta que, para cada pregunta de tipo abierto $(1,2,3,5,7,8)$, cada uno de los encuestados podía proporcionar hasta tres respuestas.

En la tabla 1 se recogen las respuestas a las preguntas $1,2,3$ y 5 ; todas ellas de tipo abierto, y se constata que respecto a la pregunta 1 , la relación legal de los informados con el paciente es más valorada que la relación afectiva, mientras que el nivel cultural y el conocimiento de la gravedad real del caso son los dos aspectos que despiertan el interés del informador en segundo término.

En lo referente a la pregunta 2, el conocer qué idea de pronóstico a corto y medio plazo se ha proporcionado a los familiares y allegados en informaciones previas es del mayor interés, así como la exhaustividad de la información proporcionada. Un número importante de encuestados (40\%) considera que dispo- 
TABLA 1. Número de respuestas obtenidas por pregunta (los encuestados podían dar hasta tres respuestas a cada pregunta abierta)

\begin{tabular}{|c|c|}
\hline Pregunta & N..$^{\circ}$ respuestas \\
\hline \multicolumn{2}{|l|}{ 1. ¿Qué quisieras saber sobre familia y allegados? } \\
\hline Relación legal con el paciente & 11 \\
\hline Conocimiento o conciencia de la gravedad real & 7 \\
\hline Nivel cultural/intelectual de los informados & 7 \\
\hline Relación afectiva con el paciente & 6 \\
\hline Si el paciente proviene de planta & 4 \\
\hline Situación emocional & 2 \\
\hline Si existen problemas de comunicación/agresividad & 2 \\
\hline Quién recibe habitualmente la información & 2 \\
\hline Trece respuestas distintas no especificadas, cada una & 1 \\
\hline \multicolumn{2}{|l|}{ 2. ¿Qué consideras importante saber de lo dicho en informaciones previas? } \\
\hline Pronóstico a corto y medio plazo & 11 \\
\hline Planes de actuación & 7 \\
\hline Un nivel de gravedad conforme a una escala simple & 6 \\
\hline Exhaustividad de la información & 5 \\
\hline Soporte que recibe el paciente & 3 \\
\hline Posibilidades de supervivencia & 3 \\
\hline Nivel terapéutico máximo deseado ¿LET? & 3 \\
\hline Pruebas pendientes & 2 \\
\hline Patología a la que se ha dado mayor importancia & 2 \\
\hline Concreción del diagnóstico & 2 \\
\hline Nueve respuestas distintas no especificadas, cada una & 1 \\
\hline \multicolumn{2}{|l|}{ 3. ¿Qué expresiones o términos crees que deben desterrarse de la información? } \\
\hline Términos ambiguos: estable, inestable, mantenido, bien, regular, igual, etc. & 11 \\
\hline $\begin{array}{l}\text { Términos médicos: exitus, carcinoma, hemodinámica, síndrome, cateterismo, nombre de drogas, } \\
\text { parámetros de ventilación, etc. }\end{array}$ & 7 \\
\hline Ocho respuestas distintas no especificadas, cada una & \\
\hline \multicolumn{2}{|l|}{ 5. Sugiere tres niveles de información que deban ser conocidos por la familia/allegados } \\
\hline Pronóstico vital a medio/corto plazo & 7 \\
\hline Diagnóstico & 5 \\
\hline Tipo de medidas asistenciales utilizadas & 3 \\
\hline Situación actual del proceso & 3 \\
\hline Afectación orgánica & 2 \\
\hline Planes a seguir & 2 \\
\hline Veintitrés respuestas distintas no especificadas, cada una & 1 \\
\hline
\end{tabular}

ner de una escala simple y comprensible de gravedad puede ser de gran ayuda.

Las respuestas más definidas se producen respecto a la pregunta 3 , siendo todos los encuestados partidarios de abstenerse de utilizar términos ambiguos que pudieran favorecer la incertidumbre en la comprensión de los mismos, y a la vez obviar un lenguaje cientifista o profesionalizado, muy alejado del conocimiento normal de los informados.

Muy lejos de esta casi unanimidad, las sugerencias respecto a los contenidos de la información que deben estar siempre presentes hacen hincapié en el

TABLA 2. Tipo de información susceptible de ser proporcionada por enfermería (pregunta 6): la respuesta debía ser Sí/No a cada una de las opciones presentadas

\begin{tabular}{|l|c|c|}
\hline Contenido & Sí & No \\
\hline Situación del paciente & 13 & 5 \\
Tratamientos en curso & 5 & 11 \\
Gravedad subjetiva & 11 & 6 \\
Diagnóstico & 4 & 14 \\
Pronóstico & 2 & 15 \\
Planes & 8 & 9 \\
Cuidados & 15 & - \\
\hline
\end{tabular}

pronóstico a corto/medio plazo y al diagnóstico de la situación del enfermo.

La tabla 2 demuestra cómo los encuestados (médicos en su mayoría) son partidarios de que la enfermería proporcione información pero sobre los cuidados, la gravedad subjetiva y la situación puntual, mostrándose contrarios a abordar temas como el

TABLA 3. Sugerencias para el diseño de una hoja de transmisión inter-profesional de la información proporcionada a la familia/los allegados

Favorables

Profesional que informa

Contenidos previos de la información

Nivel de gravedad/escala simple

Diagnóstico y situación actual

Tratamiento y planes de actuación

Lenguaje no medicalizado, simple y comprensible

Formato ficha

Complicaciones y afectaciones orgánicas secundarias

Desfavorables

Elaboración compleja

Cumplimentación tediosa («otro papel»)

Preferible transmisión oral

Procurar que la información sea dada siempre por el mismo profesional 
diagnóstico, el pronóstico y los tratamientos en curso, por considerar que todos ellos son propios de los médicos.

La tabla 3 recoge algunas sugerencias sobre la elaboración de una ficha que permita transmitir los contenidos de la información entre los profesionales y recoge, a la vez, algunos de los argumentos contrarios interpuestos frente a esta iniciativa.

La respuesta más difícil de tabular fue la correspondiente a la pregunta 4 . En total se obtuvieron 18 posibles escalas de gravedad/pronóstico diferentes, y el encuestado 19 no respondió. De entre las respuestas obtenidas y referidas a los 5 (gravedad decreciente) niveles solicitados predominaron: nivel 5, puede fallecer ( 3 respuestas); nivel 4 , muy grave ( 6 respuestas); nivel 3, sin cambios/estancado (4 respuestas); nivel 2, bien (3 respuestas); nivel 1, no precisa UCI/alta en las próximas horas (10 respuestas).

Dadas las características de los resultados, meramente descriptivos, no se realizó ningún análisis estadístico.

\section{DISCUSIÓN}

Al valorar el proceso de información concerniente a pacientes en situación crítica, deben considerarse dos pares de componentes diferenciados. El primero hace referencia al binomio informante/informado, y el segundo a los contenidos y forma de la propia información.

Nuestro trabajo trata de abordar este segundo aspecto, desde la perspectiva de los profesionales sanitarios (médicos y enfermeras) adscritos a una UCI polivalente.

Sin embargo, es preciso tratar algunos puntos sobre el primero de los aspectos: el binomio informador/informado.

La formación médica convencional (tanto pre como postgrado) obvia tratar las habilidades de información, y ello es aún más grave si se tiene en cuenta que el profesional en activo dedicará una parte fundamental de su tiempo a «hablar» con enfermos, sus familias y allegados, y que de su capacidad de transmitir adecuadamente lo que pretende va a depender la comprensión y aceptación de lo informado. Es un hecho, sin embargo, el vacío educacional existente en la aceptación de esta evidencia. Es más, el profesional con experiencia suele ser un autodidacta, que en ocasiones ha «aprehendido» unas ciertas actitudes por un proceso de ensayo/error, que está influido por el propio trasfondo emocional del profesional, que con frecuencia se refugia en actitudes muy profesionalizadas para evitar la propia implicación en el proceso, manteniendo una distancia que podríamos calificar de «estanislavsquiana» (K. Stanislavsky fue un actor y director de escena ruso [Moscú, 1863- ib. 1938], padre del teatro moderno y creador de un método interpretativo en el que se aconseja la integración del actor con el personaje, a la vez que se defiende un distanciamiento del hecho interpretativo en sí mismo).

Es frecuente que el profesional interesado en estos aspectos deba recurrir a la autoformación o a cursos complementarios, impartidos generalmente por psicólogos, que le permitan llenar este hueco en su propio conocimiento 5 .

La segunda parte de este primer binomio, el informado, es el aspecto que ha sido, tradicionalmente, más frecuentemente tratado en la bibliografía de la especialidad $^{6-10}$.

Nuestra intención ha sido, empero, centrarnos en al análisis y modificación (si fuera preciso) de los componentes del segundo binomio. El «qué»se informa y «cómo» se hace. Y ello ha sido abordado de una forma conjunta.

El contenido de la información lo constituye, obligadamente, la situación del paciente, en un sentido exhaustivamente médico y asistencial. El diagnóstico, la situación actual, las perspectivas, los planes, el tratamiento y sus posibles alternativas y el pronóstico. Ahora bien, este contenido, este «qué» debe ser ofrecido de forma tal, que el receptor lo comprenda, y pasándolo por su propio filtro, intelectual y emocional, lo asuma. Y para ello debe ser proporcionado de una forma determinada.

La teoría de la información defiende que ésta debe ser veraz, fehaciente, contrastable, suficiente, no innecesariamente alarmista y no conscientemente tenden$\operatorname{ciosa}^{11-14}$. El receptor ha de poder establecer una línea de actuación razonable en función de la información recibida, y ello será más difícil cuanto menos comprensible, más alambicado y más tecnificado sea el lenguaje utilizado. Esto se refleja en referencias que han tratado de analizar la satisfacción de los pacientes y sus allegados, respecto a la información recibida ${ }^{15,16}$, y se acentúa marcadamente en los casos asociados al fallecimiento del paciente ${ }^{17}$. Podríamos decir que cuanto más grave es la situación del enfermo, más acuciante es encontrar una forma de comunicación que resulte lo más adecuada y clara posible.

Es llamativo que nuestro estudio constate la importancia de armonizar el lenguaje de forma tal que, además de su comprensión, se evite el penoso efecto que sobre los informados tiene la detección (real o aparente) de contradicciones conforme a los distintos informantes ${ }^{18}$. Dado que las exigencias laborales impiden que la información sobre un mismo paciente pueda ser proporcionada siempre y de forma exclusiva por un único profesional, es fundamental establecer una forma de transmitir entre profesionales una información adecuada sobre lo que se ha informado por los otros posibles implicados.

Queda por describir cuáles fueron las consecuencias de nuestro estudio.

Nuestra UCI dispone desde el año 2000 de un tríptico informativo donde se describe la situación física de los cubículos (plano), la situación de los despachos de información, las normas recomendadas acerca del comportamiento durante las visitas, y la relación de profesionales médicos del servicio, así como los nombres del jefe de servicio, la supervisora de enfermería, y los teléfonos de contacto. Este tríptico se entrega personal e individualmente, en mano, a todas las familias al ingreso del paciente y cuando se nos solicita. 
APÉNDICE 1. Propuesta de hoja de transmisión de información

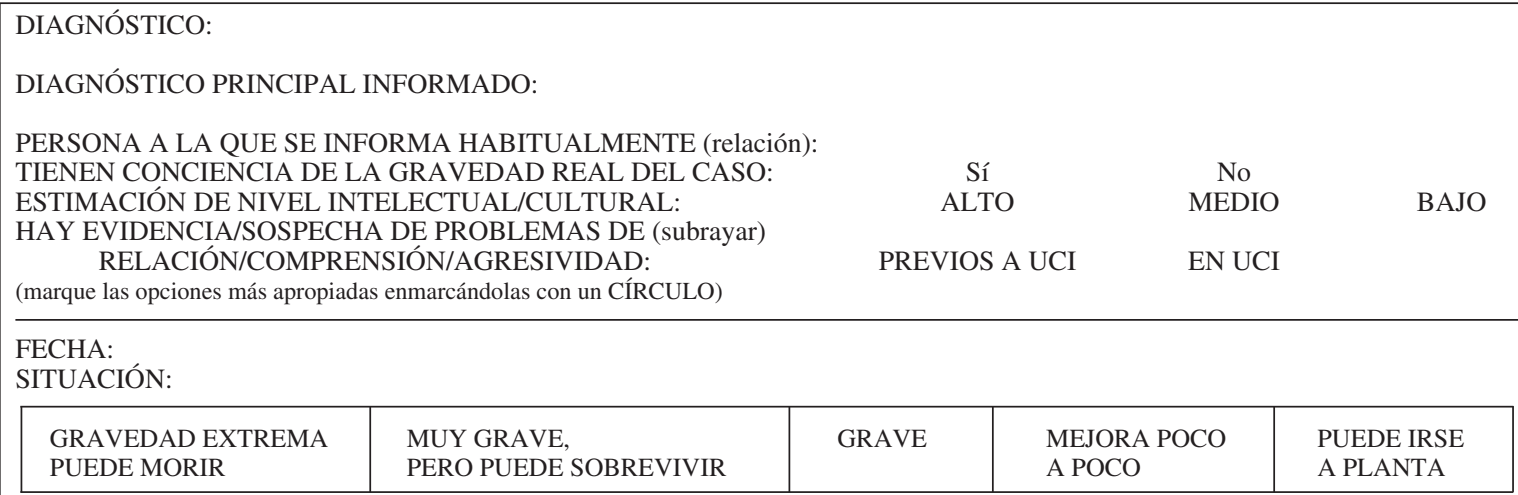

SITUACION ACTUAL (estado del proceso principal y de las complicaciones existentes):

PLANES A SEGUIR:

PRONÓSTICO VITAL (a corto/medio plazo): MALO INDETERMINADO BUENO

\section{INSTRUCCIONES GENÉRICAS}

1. La cumplimentación de esta hoja debe realizarse de forma sencilla, evitando terminología médica/profesional o términos ambiguos como: igual, regular, estable, etc.

2. El diagnóstico fundamental es para uso del personal del Servicio de Medicina Intensiva (SMI), el diagnóstico informado debería ser la "traducción" a un lenguaje normal de la misma situación; así: cardiopatía isquémica (angina frente a infarto) debería ser comunicado como "probable infarto" (a descartar o confirmar).

3. Cada día que sea necesario, se modificará (indicando la fecha) el contenido de las casillas correspondientes.

4. La situación actual y las complicaciones del proceso principal deben incluir una mención al soporte instrumental (respirador, diálisis, etc.) de los órganos en fallo. No debe hablarse de fármacos y mucho menos de sus dosis. Por ejemplo: sedantes, sin especificar tipo ni dosis.

5. Entre los planes a seguir debe figurar todo aquello que represente estrategias diagnósticas o terapéuticas que se aparten del manejo habitual rutinario en la Unidad de Cuidados Intensivos (UCI): tomografía axial computarizada (TAC), resonancia magnética nuclear $(\mathrm{RMN})$, biopsia, intervención, etc.

6. El pronóstico sólo debe tener tres posibilidades: MALO, YA VEREMOS, BUENO.

7. Enfermería puede y debe participar del hecho de la información.

La enfermera que atienda al paciente, si es interrogada por la familia o allegados DEBE RESPONDER:

a) Sobre cuidados básicos.

b) Sobre la actitud ante el enfermo (tocar, besar, hablar, ...).

c) Sobre el diagnóstico principal del enfermo (ver la hoja).

d) Sobre los planes a seguir (traslados, TAC, etc.).

e) Sobre la gravedad, utilizando la misma escala que utilizan los médicos

NO DEBE RESPONDER NI DAR INFORMACIÓN sobre:

- Pronóstico.

- Situación real actual.

8. La familia y los allegados, serán siempre invitados y estimulados a participar de los cuidados básicos del paciente, sobre todo si está consciente (comida asistida, aseo personal, movilizaciones, etc.).

9. En TODO CASO Y POR PARTE DE TODO EL MUNDO SE EVITARÁ, ESPECIALMENTE, EL USO DE PALABRAS, TÉRMINOS, EXPRESIONES, ETC., QUE SEAN DEMASIADO PROFESIONALES O DEMASIADO AMBIGUAS Y CONFUSAS.

10. La ubicación de esta hoja será la carpeta de documentación clínica, la primera hora, por delante de cualquier otro documento, y las de fecha más reciente (si hubieran varias) en primer lugar.

A partir de la elaboración de propuestas nacidas de la encuesta descrita, se confeccionó una hoja de información (situada en plafón en la sala de familiares y a la entrada de la UCI) en la que se describen las características del trabajo asistencial en pacientes críticos. También se elaboró una ficha resumen de transmisión de la información proporcionada (Apéndice 1), que debe ser cumplimentada por cada uno de los médicos después de la sesión informativa de cada día, lo que proporciona a las distintas guardias (horarios nocturnos y festivos) un instrumento sumamente útil para poder enfrentarse a familiares y allegados con los que, posiblemente, no habían tenido contacto previo. Se ha facilitado el acceso de las familias a la información a través de miembros del voluntariado del hospital, que no sólo les acompañan al despacho de información, sino que les proporcionan el nombre del médico que informa, y a éste, el del paciente familiar de los informados, aclarándoles cuantas dudas puedan tener sobre el funcionamiento de las rutinas de información.

Como conclusión, debemos decir que cada unidad debe someter sus rutinas informativas a análisis y crítica $^{19}$, con el fin de poder introducir aquellos cambios que periódicamente se consideren oportunos. 


\section{AGRADECIMIENTOS}

Los autores desean agradecer su interés y colaboración a todos los miembros del equipo de Enfermería (DUE y Auxiliares de Enfermería) adscrito al Servicio de Medicina Interna del Hospital General de Castellón.

\section{Declaración de conflicto de intereses}

Los autores han declarado no tener ningún conflicto de intereses.

\section{BIBLIOGRAFÍA}

1. http://buscon.rae.es/draeI/SrvltConsulta?TIPO_BUS=3\& LEMA=informar (consultado mayo 2007).

2. http://www.unileon.es/ficheros/investigacion/141_2002_14 nov.pdf (consultado mayo 2007).

3. Bernat Adell A, Tejedor López R, Sanchís Muñoz J. ¿Cómo valoran y comprenden los familiares la información proporcionada en una Unidad de Cuidados Intensivos? Enfermería Intensiva. $2000 ; 11: 3-9$

4. Abizanda R, Miravet V, Teruel C, Heras A, Alcala-Zamora J. Participación del enfermo crítico en la toma de decisiones. En Net A, editor. Ética y costes. Barcelona: Springer Verlag Ibérica; 1996. p. 214--226.

5. Buckman R, Korsch B, Baile W. A practical guide to communication skills in clinical practice. Toronto: Medical Audiovisual Communications; 1998.

6. Novaes MAFP, Andreoli PBA, Karam CH, Barreto AJ, Cendoroglo M, et al. Needs of families of critically ill patients and perceptions of thee health care team. Disponible en: http://ccforum.com/content/5S3/P77 (consultado mayo 2007).
7. Azoulay E, Pochard F, Chevret S, Lemaire F, Mokhtari F, et al. Meeting the needs of intensive care unit patient families. Am J Respir Crit Care Med. 2001;163:135-139.

8. Rego Lins Fumis R, Nobuko Nishimoto I, Deheinzelin D. Measuring satisfaction in family members of critically ill cancer patients in Brazil. Intens Care Med. 2006;32:124-8.

9. Azolulay E, Pochard F, Chevret S, Jourdain M, Bornstain C, Wemet A, et al. Impact of a family information leaflet on effectiveness of information provided to family members of intensive care unit patients. Am J Respi Crit Care Med. 2002;165:438 -42.

10. Alvarez GF, Kirby AS. The perspective of families of the critically ill patients: their needs. Curr Opin Crit Care. 2006;12: 614-618.

11. Bensing JM. Doctor-patient communication and the quality of care. Social Medicine. 1991;32:1301-10.

12. Campbell ML. Breaking bad news to patients. JAMA. 1994; 2071:1052-7.

13. Kennedy P, Llewelyn S. The person's experience of health care. En: Llewelyn S, Kennedy P, editors. Handbook of clinical health psychology. West Sussex: John Wiley \& Sons; 2003. p. 21-40.

14. Ong LM, Dehaes JC, Hoos AM, Lammes FB. Doctor-patient communication. A review of the literature. Social Science and Medicine. 1995;40:903-18.

15. Malacrida R, Bettelini CM, Degrate A, Martínez M, Badía F, Piazza J, et al. Reasons for dissatisfaction: a survey of relatives of intensive care who died. Crit Care Med. 1998;26:1187-1193.

16. Cuthbertson SJ, Margetts MA, Streat SJ. Bereavement follow-up after critical illness. Crit Care Med. 2000;28:1196-201.

17. Abbott KH, Sago JC, Breen CM, Abernethy AP, Tulsky JA. Families looking back: one year alter discussion of withdrawal or withholding of life sustaining report. Crit Care Med. 2001;29:217-9.

18. Moreau D, Goldgran-Toledano D, Alberti C, Jourdan M, Adrie C, Annane D, et al. Junior versus senior physicians for informing families of intensive care unit patients. Am J Respir Crit Care Med. 2004:169:512-17.

19. Azoulay E, Sprung CL. Family-physician interactions in the intensive care unit. Crit Care Med. 2004;32:2323-8. 